\title{
VARIACIÓN ALTITUDINAL EN EMERGENCIA Y CRECIMIENTO INICIAL DE PLANTAS DE Pinus patula
}

\section{ALTITUDINAL VARIATION IN EMERGENCE AND INITIAL GROWTH OF Pinus patula PLANTS}

\author{
Sandra L. Fuentes-Amaro ${ }^{1}$, Rodrigo Rodríguez-Laguna ${ }^{1}$, \\ Ramón Razo-Zárate ${ }^{1}$, Joel Meza-Rangel ${ }^{1}$ y Marcos Jiménez-Casas ${ }^{2}$
}

\begin{abstract}
'Universidad Autónoma del Estado de Hidalgo, Instituto de Ciencias Agropecuarias, Tulancingo, Hidalgo, México. ${ }^{2}$ Colegio de Postgraduados, Posgrado en Ciencias Forestales, Campus Montecillo, Montecillo, Texcoco, Estado de México, México.

*Autor de correspondencia (rlaguna@uaeh.edu.mx)
\end{abstract}

\section{RESUMEN}

Pinus patula es una especie de amplio uso e importancia en México y otros países por sus características de crecimiento y calidad de madera. Con la finalidad de identificar la existencia de un patrón de variación entre procedencias de diferente origen altitudinal (1959 a 2579 msnm), se evaluó en vivero la emergencia de plántulas, además de la altura y diámetro basal a los seis meses de edad en plantas de siete poblaciones de los estados de Hidalgo, Puebla y Veracruz, México. Se recolectó semilla de 49 árboles y la siembra se realizó en contenedores de polietileno. La emergencia de plántulas varió entre las poblaciones de 54.2 a $88.3 \%$, la velocidad de emergencia de 35.5 a 45.3 días, la altura varió de 25.8 a $27.9 \mathrm{~cm}$ y el diámetro basal de 3.4 a $3.6 \mathrm{~mm}$. Las plantas provenientes de sitios de mayor altitud tuvieron mayor porcentaje y velocidad de emergencia que las procedentes de sitios de menor elevación. No se encontró un patrón de diferenciación en el crecimiento en altura y diámetro de las plantas en relación con la altitud del sitio de origen.

Palabras clave: Pinus patula, emergencia, patrón de variación, velocidad de emergencia.

\section{SUMMARY}

Pinus patula is a widely used and very important species in México and other countries due to its growth and wood quality traits. In order to identify the existence of a variation pattern between provenances of different altitudinal origin (1959 to 2579 masl), the emergence of seedlings, as well as height and basal diameter at six months of age were evaluated in the nursery in seven populations from the states of Hidalgo, Puebla and Veracruz, Mexico. Seed was collected from 49 mother trees and sown in polyethylene containers. Seedling emergence varied between populations from 54.2 to 88.3 $\%$, the emergence speed from 35.5 to 45.3 days, seedling height from 25.8 to $27.9 \mathrm{~cm}$ and basal diameter from 3.4 to $3.6 \mathrm{~mm}$. Plants from higher altitude sites had a higher percentage and speed of emergence than those from lower elevation sites. No differentiation pattern was o found in the growth in height and diameter of plants in relation to the altitude of the provenance site.

Index words: Pinus patula, emergence, emergence speed, variation pattern

\section{INTRODUCCIÓN}

Pinus patula Schiede ex Schltdl. et Cham. es una conífera nativa de México, con amplia distribución en la Sierra Madre Oriental, principalmente en los estados de Hidalgo, Veracruz, Puebla, Oaxaca y Tlaxcala, a través de un extenso gradiente altitudinal que va de 1600 a 3100 msnm. (Nyoka, 2002; Perry, 1991). Dado que la especie se desarrolla en un amplio intervalo altitudinal, es posible que a través de procesos evolutivos se hayan conformado poblaciones en sintonía con el ambiente local y, por ende, haya diferenciación genética entre ellas (Rehfeldt, 1993).

La emergencia de la plántula es crucial en la adaptación de las plantas porque establece el contexto para el desarrollo posterior; además, las condiciones ambientales que estimulan la emergencia y el crecimiento inicial de la planta son aquellas que el nuevo individuo debe afrontar (Donohue et al., 2010). En parámetros germinativos, la variación dentro de la especie generalmente se asocia con diferencias en las condiciones del sitio (Baskin y Baskin, 2001).

Para identificar patrones de variación se evalúan características adaptativas y se relacionan con características geográficas o climáticas del sitio de origen (Castellanos-Acuña et al., 2013; Martínez-Berdeja et al., 2019). Uno de los factores geográficos con los que más se han relacionado los patrones de variación en las coníferas es la altitud (Sáenz-Romero, 2011). Para el manejo y conservación de los recursos genéticos se requiere conocer los patrones de variación genética intraespecífica (Furnier, 2004). Si existe un patrón de variación genética dentro del área de distribución natural de la especie se recomienda definir lineamientos para el movimiento de germoplasma, con la finalidad de que exista acoplamiento 
entre los genotipos y, como resultado, las reforestaciones sean exitosas (Sáenz-Romero et al., 2006; White et al., 2007).

Por lo anterior, se han estudiado los patrones de variación en especies forestales utilizadas en los programas de reforestación (Sáenz-Romero et al., 2011). En procedencias de P. patula del sur de Oaxaca se identificó que las poblaciones de altitud intermedia (2650 msnm) mostraron el mejor crecimiento en altura (Ruiz-Talonia et al., 2014) en comparación con las poblaciones de los extremos altitudinales. Para las poblaciones de P. patula de la región centro de su distribución no se conocen los patrones de variación genética.

Por la razón anterior, se planteó el objetivo de determinar en vivero la existencia de diferencias en características de emergencia y crecimiento inicial de las plantas en siete poblaciones de P. patula y de relacionar el patrón de variación de la emergencia y crecimiento inicial de las plantas con la altitud. De acuerdo con las diferentes condiciones ambientales en las que se desarrolla la especie, se espera que existan diferencias entre las poblaciones y que dichas diferencias se relacionen con la altitud del sitio de origen.

\section{MATERIALES Y MÉTODOS}

\section{Recolección de semilla}

La semilla se recolectó en siete poblaciones localizadas en el área centro de la distribución natural de la especie en México (Cuadro 1). La recolecta se realizó en árboles sanos, de fuste recto, con altura y diámetro superior al promedio del rodal. Se seleccionaron de cinco a ocho árboles en cada localidad, con un total de 49 individuos en las siete poblaciones. Los árboles estuvieron a distancias mayores de $100 \mathrm{~m}$ para evitar recolectar árboles emparentados. A través de escalado se obtuvieron conos de polinización abierta de cada uno de los árboles en mayo y junio de 2018. Las muestras se identificaron y transportaron al Laboratorio de Semillas y Germoplasma del Instituto de Ciencias Agropecuarias de la Universidad Autónoma del Estado de Hidalgo, en Tulancingo, Hidalgo, México. En este lugar se llevó a cabo el beneficio y almacenamiento de la semilla hasta su uso en el ensayo de germinación y crecimiento de las plantas.

En lo sucesivo, el término "procedencia" se utilizará para referirse a la localidad de origen y "población" para referirse a la progenie derivada del conjunto de individuos de cada procedencia.

\section{Siembra}

Lamezcla de sustratos para la siembra estuvo compuesta por $60 \%$ aserrín, $15 \%$ turba de musgo, $15 \%$ tezontle y $10 \%$ vermiculita, más $8 \mathrm{~g} \mathrm{~L}^{-1}$ de fertilizante de liberación controlada de ocho meses (12-09-16). Las semillas se sembraron en tubos rígidos de polietileno negro de 310 $\mathrm{cm}^{3}$ en diciembre de 2019, en condiciones de invernadero. La profundidad de siembra fue de aproximadamente $1 \mathrm{~cm}$, para lo que se utilizó una vareta marcada que se sumergió en cada uno de los tubos. En los días subsecuentes a la siembra el riego fue diario y al emerger las plantas se regó cada tercer día.

\section{Diseño experimental}

El ensayo se estableció en invernadero, bajo un diseño de bloques completos al azar con tres repeticiones. Después de emerger, las plántulas se llevaron a una casa sombra, con el mismo diseño de bloques completos al azar, con tres repeticiones y parcelas experimentales de 15 plantas por familia.

Cuadro 1. Localidades y número de árboles de $P$. patula seleccionados para la recolecta.

\begin{tabular}{lllcc}
\hline Localidad & Municipio & Entidad & Número de árboles & Altitud $(\mathrm{msnm})$ \\
\hline Xonocuautla & Tlatlahuquitepec & Puebla & 8 & 2579 \\
Ojo de Agua & Huayacocotla & Veracruz & 8 & 2532 \\
Cumbre de Muridores & San Bartolo & Hidalgo & 5 & 2361 \\
Ejido Beristain & Ahuazotepec & Puebla & 8 & 2342 \\
La Cruz Verde & Metztitlán & Hidalgo & 5 & 2136 \\
El Reparo & Zacualtipan & Hidalgo & 7 & 2068 \\
La Selva & Huayacocotla & Veracruz & 8 & 1959 \\
Total & & 49 & \\
\hline
\end{tabular}




\section{Variables evaluadas}

Se consideró plántula emergida cuando los cotiledones fueron visibles por encima de la superficie (Mukherjee Roy et al., 2004). El conteo de las plántulas que emergieron se hizo cada tres días, comenzando desde el día 30 (a partir de la siembra), que fue cuando emergieron las primeras plántulas. El porcentaje de plantas emergidas se definió como la proporción de plantas que emergió con respecto al total de semilla sembrada en cada parcela. La variable "tiempo medio de emerger" $\left(T_{50}\right)$ se definió como el día en que se alcanzó 50 \% de plantas emergidas del total de semilla sembrada, como una medida de la velocidad de aparición de las plántulas. A los seis meses de edad (a partir de que emergieron las plántulas) se midió la altura de la parte aérea y el diámetro en la base del tallo. La altura se midió en $\mathrm{cm}$ con una regla graduada y el diámetro en $\mathrm{mm}$ con un vernier (Mitutoyo ${ }^{\circledR}$, Kanagawa, Japón). El número de plantas, que se midió por población, varió de 225 a 360, dependiendo del número de familias que se incluyó en cada una de ellas (Cuadro 1). Para las variables de crecimiento se obtuvieron valores promedio por parcela para cada procedencia, previo al análisis estadístico.

\section{Análisis estadístico}

Para determinar las diferencias entre las procedencias se realizó un análisis de varianza (ANOVA) con el procedimiento GLM del programa estadístico SAS (Statistical Analysis System) Ver. 9.0 (SAS Institute, 2002). En todas las variables se utilizó el modelo estadístico siguiente, con los valores promedio por parcela:

$$
Y_{i j}=\mu+B_{i}+P_{j}+E_{i j}
$$

donde: $Y_{i j}$ es el valor de la característica de la j-ésima población en el i-ésimo bloque, $\mu$ es la media poblacional, $B_{i}$ es el efecto del i-ésimo bloque, $P_{j}$ es el efecto fijo de la j-ésima procedencia y $E_{i j}$ es el error experimental.

Para identificar el patrón altitudinal de variación del porcentaje y velocidad de emergencia de las plantas, así como de la altura y diámetro de las plantas a los seis meses de edad con respecto a la altitud del sitio de origen, se realizó un análisis de regresión lineal con el procedimiento REG de SAS (SAS Institute, 2002) de los valores medios de las poblaciones con respecto a la altitud de origen, con el siguiente modelo:

$$
Y_{i}=\beta_{0}+B_{7} X_{i}+\varepsilon_{i}
$$

donde: $Y_{i}$ es el valor medio de la i-ésima población, $\beta_{0}$ es el intercepto, $B_{7}$ es el coeficiente de regresión, $X_{i}$ es la altitud de la i-ésima procedencia y $\varepsilon_{i}$ es el error aleatorio.
La variable porcentaje de plántulas emergidas se transformó con la función arcoseno de la raíz cuadrada de los datos originales divididos entre 100 porque no cumplía con el supuesto de normalidad.

\section{RESULTADOS Y DISCUSIÓN}

\section{Diferencias en emergencia y crecimiento}

Se encontraron diferencias significativas $(P \leq 0.05)$ entre las poblaciones en las características de emergencia de las plántulas (Cuadro 2). Para otras especies como Nothofagus glauca (Phil.) (Santelices et al., 2017) y Pinus greggii Engelm (Mendizábal-Hernández et al., 2015) también se han reportado diferencias tanto en porcentaje como en velocidad de emergencia de las plántulas.

Las plántulas empezaron a emerger a los 30 días después de la siembra (Figura 1), con excepción de las semillas de La Cruz Verde, El Reparo y La Selva, que iniciaron a los 33 días. Las plantas de Xonocuautla, Ojo de Agua, Cumbre de Muridores y Ejido Beristain emergieron más uniformemente, ya que completaron, en promedio, $62 \%$ de plantas emergidas a los 45 días después de la siembra; por el contrario, las semillas de La Cruz Verde, El Reparo y La Selva alcanzaron $38.3 \%$ de plantas emergidas en esta fecha. A partir del día 48, la curva para cada una de las poblaciones comenzó a estabilizarse, con excepción de la de La Selva.

El promedio de plántulas emergidas fue $67.9 \%$ (Cuadro 2). Fuentes-Amaro et al. (2020) evaluaron las plantas emergidas en las poblaciones de Ojo de Agua y Cumbre de Muridores y encontraron 86.1 \% en promedio. Si se consideraran únicamente estas dos poblaciones en el presente estudio, el promedio sería similar (83.3\%).

En condiciones naturales del bosque, las plántulas emergen de la semilla en respuesta al aumento de la temperatura y humedad (Ramírez et al., 2015) y el momento en que se presenta es crucial porque puede tener efectos en la supervivencia y crecimiento inicial de las plantas (Verdú y Traveset, 2005).

El tiempo promedio para obtener $50 \%$ de plantas emergidas fue de 39.3 días, valor alto en comparación con el obtenido por Fuentes-Amaro et al. (2020) en poblaciones de P. patula. Entre estos dos experimentos hubo una diferencia de 15 días en el inicio de aparición de las plantas. Considerando que el método utilizado fue similar, tales diferencias se explican por las condiciones ambientales prevalecientes en los días subsecuentes a la siembra. En el presente estudio la siembra se realizó en el mes de diciembre de 2018, en el que predominan 


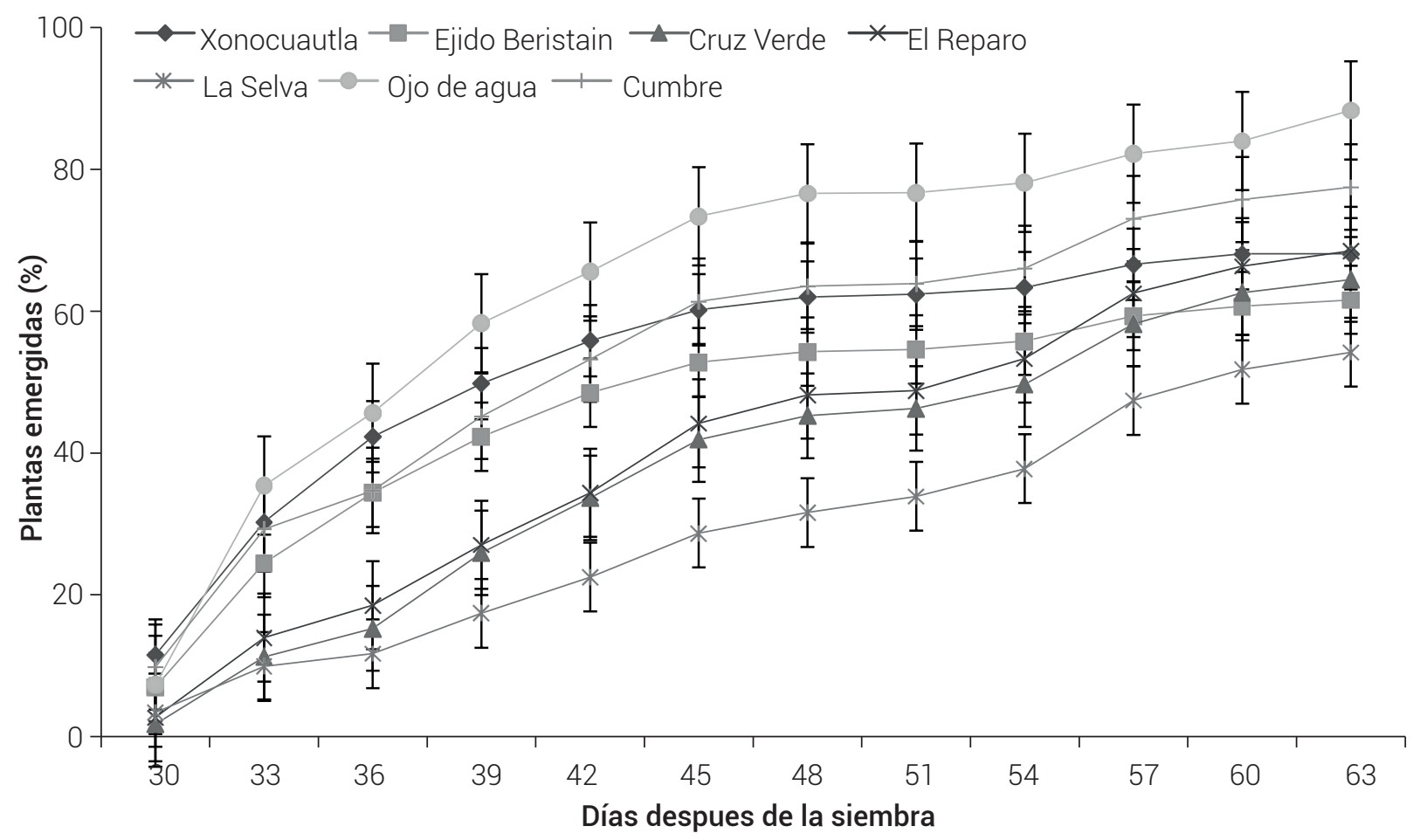

Figura 1. Curva acumulativa de plantas emergidas de semillas de siete poblaciones naturales de $P$. patula.

Cuadro 2. Media, error estándar y prueba de Tukey para el porcentaje y velocidad de plantas emergidas $\left(T_{50}\right)$ y la altura y diámetro del tallo en plantas de seis meses de edad de poblaciones naturales de $P$. patula.

\begin{tabular}{|c|c|c|c|c|}
\hline Población & Plantas emergidas (\%) & $\mathrm{T}_{50}$ (días) & Altura $(\mathrm{cm})$ & Diámetro $(\mathrm{mm})$ \\
\hline Xonocuautla & $68.1 \pm 1.83 \mathrm{bc}$ & $35.4 \pm 0.60 b$ & $26.5 \pm 0.19 \mathrm{~cd}$ & $3.5 \pm 0.03 \mathrm{ab}$ \\
\hline Ojo de Agua & $88.3 \pm 2.61 \mathrm{a}$ & $36.0 \pm 0.63 b$ & $27.9 \pm 0.20 a$ & $3.6 \pm 0.03 a$ \\
\hline Cumbre & $77.7 \pm 2.09 a b$ & $37.6 \pm 1.03 b$ & $27.0 \pm 0.28 \mathrm{abc}$ & $3.5 \pm 0.05 a b$ \\
\hline Ahuazotepec & $61.6 \pm 3.97 \mathrm{bc}$ & $36.4 \pm 0.58 b$ & $26.7 \pm 0.21 \mathrm{bcd}$ & $3.5 \pm 0.03 a b$ \\
\hline Cruz Verde & $55.2 \pm 7.04 \mathrm{c}$ & $41.6 \pm 1.64 \mathrm{a}$ & $27.5 \pm 0.26 a b$ & $3.5 \pm 0.04 \mathrm{ab}$ \\
\hline El Reparo & $69.1 \pm 3.86 \mathrm{bc}$ & $42.8 \pm 0.98 a$ & $25.8 \pm 0.23 d$ & $3.4 \pm 0.03 b$ \\
\hline La Selva & $54.2 \pm 3.50 c$ & $45.3 \pm 1.52 \mathrm{a}$ & $26.7 \pm 0.20 \mathrm{bcd}$ & $3.6 \pm 0.02 a$ \\
\hline
\end{tabular}


temperaturas bajas, por lo que las plántulas comenzaron a emerger hasta que aumentó la temperatura, a finales de enero del siguiente año.

Se identificó un patrón altitudinal lineal para el porcentaje de plantas emergidas $\left(R^{2}=0.44, P=0.1\right)$ y su velocidad, evaluada en términos de $T_{50}\left(R^{2}=0.93, P=0.0004\right)$ (Figura 2), donde las poblaciones de mayor altitud producen semillas con mayor porcentaje y velocidad de germinación en comparación con las poblaciones de menor elevación. En Betula papyrifera Marsh. (Benowicz et al., 2001) se encontró un patrón semejante, en el que las poblaciones de mayor elevación produjeron semilla con mayor capacidad y velocidad germinativa.

De acuerdo con las condiciones del sitio de origen de las poblaciones, la velocidad con la que emergen puede tener beneficios para el establecimiento y crecimiento inicial de las plántulas (Baskin y Baskin, 2001); por ejemplo, la aparición lenta de las plantas debido a una baja velocidad de germinación (como es el caso de las poblaciones de La Cruz Verde, El Reparo y La Selva) podría reducir la exposición de las plántulas a eventos de mortalidad aleatorios como una helada (Mercer et al., 2011).

La altura promedio de las plantas fue $26.8 \mathrm{~cm}$. La diferencia entre los valores extremos (El Reparo y Ojo de Agua) fue de $8 \%$. El diámetro promedio fue $3.5 \mathrm{~mm}$ y la diferencia entre el mayor y el menor valor fue de sólo 5 $\%$. En algunos casos, la aparición rápida de las plantas se relaciona con mayor crecimiento inicial (Parker et al., 2006; Xu et al., 2016), como sucedió con las plántulas de Ojo de Agua; sin embargo, las plantas de otras poblaciones que también emergieron con mayor velocidad no mostraron crecimiento sobresaliente (Xonocuautla); por el contrario, las plantas de La Cruz Verde, que emergieron más lento, mostraron mayor tamaño al final del estudio. Factores como el tamaño de la semilla también puede repercutir en el desarrollo inicial de las plántulas (Xu et al., 2016); no obstante, dado que en este estudio no se consideró dicha variable, no se puede estimar la relación entre esas características.

En las variables altura $\left(R^{2}=0.12, P=0.43\right)$ y diámetro $\left(R^{2}=0.02, P=0.75\right)$ del tallo a los seis meses de edad no se identificó un patrón de diferenciación con respecto a la altitud del sitio de origen. Aunque en algunas especies se han reportado diferencias en crecimiento inicial (en vivero) relacionadas con la altitud de origen (Castellanos-Acuña et al., 2013); en otros casos, el crecimiento inicial está relacionado en mayor medida con el tamaño de la semilla (Xu et al., 2016); por ejemplo, en poblaciones de P. patula de Ixtlán, Oaxaca, México tampoco se encontró un patrón definido entre el crecimiento en altura y la altitud en plantas de seis meses de edad en vivero (Sáenz-Romero et al., 2011). No obstante, Ruiz-Talonia et al. (2014) encontraron a los 18 meses de edad, en campo, un patrón definido de diferenciación con respecto a la altitud.

\section{CONCLUSIONES}

Existen diferencias entre las poblaciones de P. patula en el porcentaje y velocidad de emergencia de plántulas, así como en el crecimiento inicial en altura y diámetro del tallo en vivero. Las poblaciones ubicadas a mayor elevación produjeron semilla con mayor porcentaje de
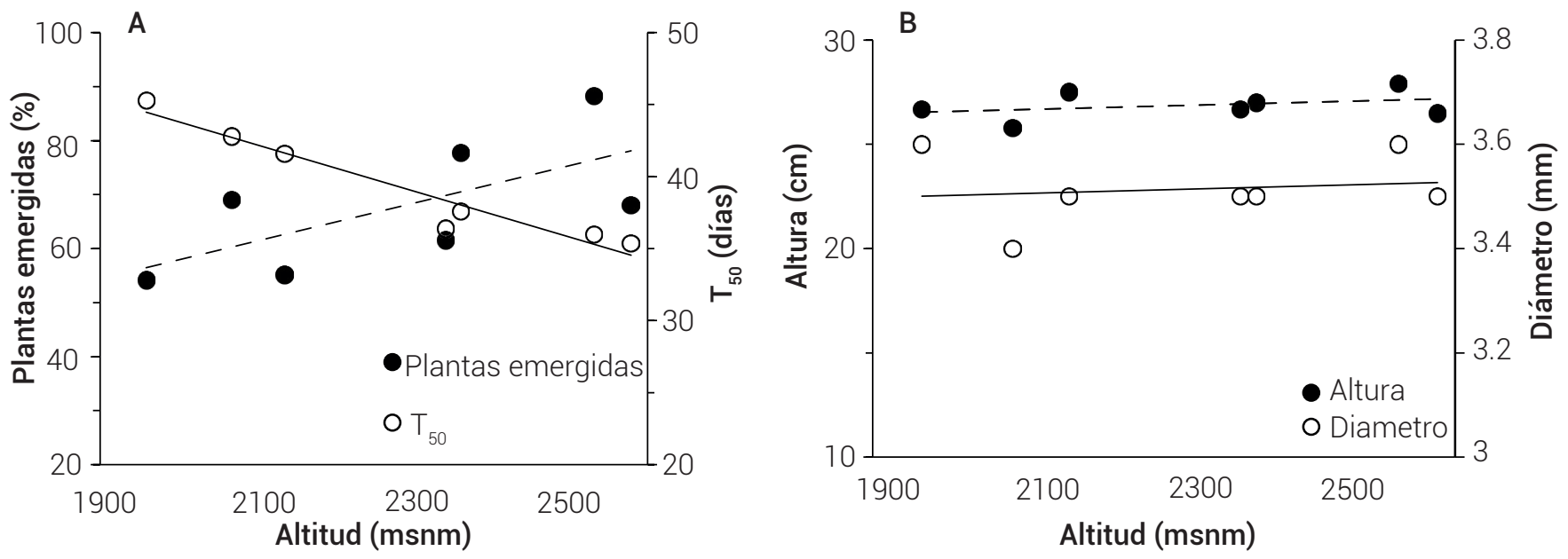

Figura 2. Relación de A) porcentaje y velocidad $\left(T_{50}\right)$ de plantas emergidas, y $B$ ) altura y diámetro del tallo a los seis meses de edad en plantas de siete poblaciones naturales de $P$. patula con la altitud del sitio de origen. 
emergencia mayor y velocidad de plantas emergidas en comparación con las poblaciones de menor altitud. Las variables de crecimiento en altura y diámetro no presentaron un patrón definido con respecto a la altitud de origen de las poblaciones. Los resultados obtenidos son útiles para planear la producción de planta en vivero y podrían ser considerados al establecer lineamientos para el movimiento de la semilla o de las plantas de estas poblaciones fuera de su sitio de origen.

\section{AGRADECIMIENTOS}

Este trabajo se derivó del proyecto "Establecimiento de huertos semilleros asexuales regionales y ensayos de progenie de Pinus patula para la evaluación genética de los progenitores" financiado a través del fondo sectorial para la investigación, el desarrollo y la innovación tecnológica forestal CONACYT-CONAFOR. También agradecemos a los representantes de los ejidos que permitieron la colecta del material vegetal.

\section{BIBLIOGRAFÍA}

Baskin C. C. and J. M. Baskin (2001) Seeds: Ecology, Biogeography, and Evolution of Dormancy and Germination. Academic Press. San Diego, California, USA. 666 p.

Benowicz A., R. Guy, M. R. Carlson and Y. A. El-Kassaby (2001) Genetic variation among paper birch (Betula papyrifera Marsh.) populations in germination, frost hardiness, gas exchange and growth. Silvae Genetica 50:7-13,

Castellanos-Acuña D., C. Sáenz-Romero, R. A. Lindig-Cisneros, N. M. SánchezVargas, P. Lobbit y J. C. Montero-Castro (2013) Variación altitudinal entre especies y procedencias de Pinus pseudostrobus, $P$. devoniana y P. leiophylla. Ensayo de vivero. Revista Chapingo Serie Ciencias Forestales y del Ambiente 3:399-411, https://doi. org/10.5154/r.rchscfa.2013.01.002

Donohue K., R. Rubio C., L. Burghardt, K. Kovach and C. G. Willis (2010) Germination, postgermination, adaptation and species ecological ranges. Annual Review of Ecology, Evolution, and Systematics 41:293-319, https://doi.org/10.1146/annurevecolsys-102209-144715

Fuentes-Amaro S. L., R. Rodríguez-Laguna, R. Razo-Zárate, J. Meza-Rangel, M. Jiménez-Casas y G. A. López Z. (2020) Energía germinativa en árboles selectos de Pinus patula Schl. et Cham. Revista Iberoamericana de Ciencias 7:69-78.

Furnier G. R. (2004) Métodos para medir variación genética en las plantas. In: Manejo de Recursos Genéticos Forestales. 2a edición. J. J. Vargas H., B. Bermejo V. y F. T. Ledig (eds.). Colegio de Postgraduados, Comisión Nacional Forestal. Montecillo, Edo. de México y Zapopan, Jalisco, México. pp:20-30.

Martínez-Berdeja A., J. A. Hamilton, A. Bontemps, J. Schmitt and J. W. Wright (2019) Evidence for population differentiation among Jeffrey and Ponderosa pines in survival, growth and phenology. Forest Ecology and Management 434:40-48, https://doi.org/10.1016/j. foreco.2018.12.009

Mendizábal-Hernández L. C., J. Alba-Landa, M. C. Rodríguez-Juárez, E. 0. Ramírez-García, J. Márquez-Ramírez y H. Cruz-Jiménez (2015) Estudio de germinación de cinco procedencias de Pinus greggii Engelm. Foresta Veracruzana 17:49-56.

Mercer K. L., H. M. Alexander and A. A. Snow (2011) Selection on seedling emergence timing and size in an annual plant, Helianthus annus (common sunflower, Asteraceae). American Journal of Botany 98:975-985, https://doi.org/10.3732/ajb.1000408

Mukherjee Roy. S., R. C. Thapliyal and S. S. Phartyal (2004) Seed source variation in cone, seed and seedling characteristic across the natural distribution of Himalayan low level pine Pinus roxburghii Sarg. Silvae Genetica 53:116-123, https://doi.org/10.1515/sg2004-0021

Nyoka B. I. (2002) Pinus patula Schiede ex Schltdl. \& Cham. In: Pines of Silvicultural Importance. D. Flinn (ed.). CABI Publising. Wallingford, UK. pp:303-316.

Parker W. C., T. L. Noland and A. E. Morneault (2006) The effects of seed mass on germination, seedling emergence, and early seedling growth of eastern white pine (Pinus strobus L.). New Forests 32:33-49, https://doi.org/10.1007/s11056-005-3391-1

Perry J. P. (1991) The Pines of México and Central America. Timber Press, Inc. Portland, Oregon. USA. 231 p.

Ramírez S. R., G. Ángeles P., R. Clark T., V. M. Cetina A., O. Plascencia E. y P. Hernández R. (2015) Efectos del manejo forestal en la repoblación de Pinus spp. en la Sierra Norte de Oaxaca, México. Revista Mexicana de Ciencias Forestales 6:49-62, https://doi. org/10.29298/rmcf.v6i32.98

Rehfeldt G. E. (1993) Genetic variation in the Ponderosae of the Southwest. American Journal of Botany 80:330-343, https:// doi.org/10.1002/j.1537-2197.1993.tb13807.x

Ruiz-Talonia L. F., N. M. Sánchez-Vargas, J. S. Bayuelo-Jiménez, S. I. LaraCabrera and C. Sáenz-Romero (2014) Altitudinal genetic variation among native Pinus patula provenances: performance in two locations, seed zone delineation and adaptation to climate change. Silvae Genetica 63:139-148, https://doi.org/10.1515/ sg-2014-0019

Sáenz-Romero C. (2011) Guía para mover altitudinalmente semillas y plantas de Pinus oocarpa, P. devoniana (= P. michoacana) P. pseudostrobus, P. patula y P. hartwegii para restauración ecológica, conservación, plantaciones comerciales y adaptación al cambio climático. Grupo de Trabajo sobre Recursos Genéticos, Comisión Forestal para América del Norte, ONU. Roma. https://www.fs.fed.us/global/nafc/ genetics/2009/SaenzRomero_2011_GuiaAltitudinal_v04.pdf (Marzo 2020).

Sáenz-Romero C., L. F. Ruiz-Talonia, J. Beaulieu, N. M. Sánchez-Vargas and G. Rehfeldt (2011) Genetic variation among Pinus patula populations along an altitudinal gradient. Two environment nursery tests. Revista Fitotecnia Mexicana 34:19-25, https:// doi.org/10.35196/rfm.2011.1.19

Sáenz-Romero C., R. R. Guzmán-Reyna and G. E. Rehfeldt (2006) Altitudinal genetic variation among Pinus oocarpa populations in Michoacán, Mexico: implications for seed zoning, conservation, tree breeding and global warming. Forest Ecology and Management 229:340-350, https://doi.org/10.1016/j. foreco.2006.04.014

Santelices M. R., S. Espinoza M., C. Magni D., A. Cabrera A., S. Donoso C. and K. Peña-Rojas (2017) Variability in seed germination and seedling growth at the intra- and inter-provenance levels of Nothofagus glauca (Lophozonia glauca), an endemic species of Central Chile. New Zealand Journal of Forestry Science 47:10, https:// doi.org/10.1186/s40490-017-0091-5

SAS Institute (2002) SAS ${ }^{\circledR} /$ STAT User's Guide. Version 9.0. SAS Institute. Cary, North Carolina. USA. 1503 p.

Verdú M. and A. Traveset (2005) Early emergence enhances plant fitness: a phylogenetically controlled meta-analysis. Ecology 86:13851394, https://doi.org/10.1890/04-1647

White T. L., W. T. Adams and B. D. Neale (2007) Forest Genetics. CAB International. Oxford, UK. 682 p.

Xu Y., N. Cai, B. He, R. Zhang, W. Zhao, J. Mao, ... and K. Woeste (2016) Germination and early seedling growth of Pinus densata Mast. provenances. Journal of Forestry Research 27:283-294 https://doi.org/10.1007/s11676-015-0186-x 